\title{
On the lcm of the Differences of Eight Primes
}

\author{
By François Morain*
}

\begin{abstract}
Following C. A. Spiro, who has found eight primes for which$$
\operatorname{lcm}\left(p_{j}-p_{i}\right)_{1 \leq i<j \leq 8}=5040,
$$

we show that for every set of eight odd primes $\left\{q_{1}, \ldots, q_{8}\right\}$, one has $5040 \mid \operatorname{lcm}\left(q_{j}-q_{i}\right)$. Moreover, $\operatorname{lcm}\left(q_{j}-q_{i}\right)=5040$ infinitely often, under the assumption of the 8-tuple conjecture.
\end{abstract}

1. Introduction. Let $Q$ be a set of eight odd primes $\left\{q_{1}, \ldots, q_{8}\right\}$ with $q_{1}<$ $\cdots<q_{8}$. We define

$$
r(Q)=\operatorname{lcm}\left(q_{j}-q_{i}\right)_{1 \leq i<j \leq 8} .
$$

In [6], C. A. Spiro proved that there are infinitely many integers $n$ for which $d(n)=$ $d(n+5040)$, where

$$
d(m)=\sum_{\delta \mid m} 1,
$$

using the fact that

$$
r(\{11,17,23,29,41,47,53,59\})=5040 .
$$

Paul Erdős has observed that for every $Q$,

$$
2520 \mid r(Q)
$$

holds, and then he conjectured that in fact $5040 \mid r(Q)$ for every $Q$.

We shall prove:

THEOREM 1. For every $Q, 5040 \mid r(Q)$.

Let $\mathscr{P}$ be a set of primes and $\mathscr{S}$ any set of integers. After Hardy and Littlewood [2] and Richards [5], we say that $\mathscr{S}$ is $\mathscr{P}$-admissible if and only if

$$
\forall p \in \mathscr{P}, \mathscr{S} \text { does not contain a complete residue system modulo } p \text {. }
$$

We then have the $k$-tuple conjecture (cf. [2]):

CONJECTURE 1. Let $0 \leq b_{1}<\cdots<b_{k}$ be integers and $\mathscr{P}=\{p$ prime $p \leq k\}$. Then, there are infinitely many integers $n$ for which all the values of $n+b_{1}, \cdots, n+b_{k}$ are prime if and only if $\left\{b_{1}, \cdots, b_{k}\right\}$ is $\mathscr{P}$-admissible.

Following Richards [5], we observe that there are two categories of octuplets of primes $Q$ :

(i) $Q$ is $\{3,5,7\}$-admissible.

Received August 18, 1987; revised November 10, 1987 and March 7, 1988.

1980 Mathematics Subject Classification (1985 Revision). Primary 11A07; Secondary 11A41.

* On leave from the French Department of Defense, Délégation Générale pour l'Armement. 
(ii) $Q$ is not $\{3,5,7\}$-admissible, but does contain 3,5 or 7 , and then $q_{1} \leq 7$. Clearly, if $Q$ is not $p$-admissible, there is a $q \in Q$ such that $q \equiv 0 \bmod p$, and this implies $q=p$.

We shall prove:

THEOREM 2. There are only four nonadmissible octuplets of primes $Q$ such that $r(Q)=5040$. There are exactly 22 different admissible sets $\left(0, b_{2}, \ldots, b_{8}\right)$ such that, if $Q=\left\{q_{1}, q_{1}+b_{2}, \ldots, q_{1}+b_{8}\right\}$ is an octuplet of primes, then we have $r(Q)=5040$.

2. Proof of (1). Let $\varphi$ be Euler's totient function. For every $m$ in $A=$ $\{5,7,8,9\}$, one has $\varphi(m) \leq 6$, and for $q$ prime, there are at most seven possible values of $q \bmod m$. By the pigeon-hole principle, we see that there exist $q$ and $q^{\prime}$ in $Q$ such that $q \equiv q^{\prime} \bmod m$, for every $m$ in $A$, thus $m \mid r(Q)$ and

$$
\prod_{m \in A} m=2520 \mid r(Q) \text {. }
$$

3. Proof of Theorem 1. Let us look for a set $Q$ for which $r(Q)=2520$. In other words,

$$
\forall 1 \leq i<j \leq 8, \quad q_{j}-q_{i} \mid 2520 .
$$

Define $a_{j}=q_{j}-q_{1}, 1 \leq j \leq 8$. We may associate with $Q$ the set $\mathscr{A}=\left\{a_{1}=\right.$ $\left.0, a_{2}, \ldots, a_{8}\right\}: Q$ is completely determined by $\left(q_{1}, \mathscr{A}\right)$. We have

$$
\forall 1 \leq i<j \leq 8, \quad a_{j}-a_{i} \mid 2520 .
$$

Since the $q_{i}$ 's are odd, the $a_{i}$ 's are even and from (4),

$$
\forall 2 \leq j \leq 8, \quad 2\left|a_{j}\right| 2520 .
$$

Let $V=\left\{v_{1}, \ldots, v_{36}\right\}$ be the set of all the even divisors of 2520 and $G=(V, E)$ be the graph of vertices $V$ and edges $E$ defined by

$$
\left(v_{i}, v_{j}\right) \in E \Leftrightarrow\left|v_{i}-v_{j}\right| \mid 2520 .
$$

Then finding the $a_{i}$ 's satisfying (4) is equivalent to searching for a complete subgraph of $G$ of seven elements, that is, a subgraph of seven elements whose vertices are pairwise adjacent. Some algorithms have been designed to solve this problem (see [1], [4]). Having implemented the algorithm given in [4, Ex. 23, p. 10], I ran the program on $G$ and found eight sets $S_{1}, \ldots, S_{8}$ that are shown in Table 1 below.

TABLE 1. S-sets

\begin{tabular}{|rrrrrrrr|}
\hline & $a_{2}$ & $a_{3}$ & $a_{4}$ & $a_{5}$ & $a_{6}$ & $a_{7}$ & $a_{8}$ \\
\hline$S_{1}$ & 2 & 4 & 6 & 8 & 10 & 12 & 14 \\
$S_{2}$ & 2 & 6 & 8 & 10 & 12 & 14 & 20 \\
$S_{3}$ & 4 & 6 & 8 & 10 & 12 & 14 & 18 \\
$S_{4}$ & 4 & 6 & 10 & 12 & 14 & 18 & 24 \\
$S_{5}$ & 6 & 8 & 10 & 12 & 14 & 18 & 20 \\
$S_{6}$ & 6 & 10 & 12 & 14 & 18 & 20 & 24 \\
$S_{7}$ & 6 & 10 & 12 & 18 & 20 & 24 & 30 \\
$S_{8}$ & 6 & 12 & 18 & 24 & 30 & 36 & 42 \\
\hline
\end{tabular}

With $\mathscr{A}_{i}=\{0\} \cup S_{i}$, we observe now that:

1. $\forall p \in\{3,5,7\}, \mathscr{A}_{1}$ is not $p$-admissible. 
2. $\mathscr{A}_{2}, \ldots, \mathscr{A}_{7}$ are just 7 -admissible.

3. $\mathscr{A}_{8}$ is just 3 -admissible.

So, $Q$ cannot belong to category (i). If $Q$ belongs to category (ii), then $q_{1} \in\{3,5,7\}$. Looking at the 24 possibilities of $\left(q_{1}, \mathscr{A}_{i}\right)$, none of them is made up of primes; this completes the proof of Theorem 1 .

4. Proof of Theorem 2. We now look for $Q$ such that $r(Q)=5040$. Following the same line of reasoning, we form $G^{\prime}=\left(V^{\prime}, E^{\prime}\right)$, where $V^{\prime}$ is the set of the 48 even divisors of 5040 and $E^{\prime}$ is defined by

$$
\left(v_{i}, v_{j}\right) \in E^{\prime} \Leftrightarrow\left|v_{i}-v_{j}\right| \mid 5040 .
$$

The computer gave 493 complete subgraphs $\mathscr{S}$ of order 7 . Only 22 of them give $\{3,5,7\}$-admissible sets $\mathscr{A}=\{0\} \cup \mathscr{S}$. These $22 \mathscr{S}$ 's are shown in Table 3 , with the smallest possible $q_{1}$. All the sets $Q$ belonging to category (i) correspond to one of these $22 \mathscr{S}$ 's, with varying $q_{1}$. Observe that C. A. Spiro's example is the fourth one.

The 471 other subgraphs can give only octuplets belonging to category (ii), with $q_{1} \leq 7$. A study of all the possible cases gave four solutions of category (ii). They are listed in Table 2.

\section{TABLE 2. Nonadmissible $S$-sets}

\begin{tabular}{|rrrrrrrr|}
\hline$q_{1}$ & $a_{2}$ & $a_{3}$ & $a_{4}$ & $a_{5}$ & $a_{6}$ & $a_{7}$ & $a_{8}$ \\
\hline 3 & 2 & 4 & 8 & 10 & 14 & 16 & 20 \\
3 & 4 & 8 & 10 & 14 & 16 & 20 & 28 \\
5 & 6 & 8 & 12 & 18 & 24 & 36 & 48 \\
5 & 6 & 12 & 18 & 24 & 36 & 42 & 48 \\
\hline
\end{tabular}

5. Generalization. Following a suggestion from the referee, we now state a more general problem. For any set $Q$ of odd primes, $Q=\left\{q_{1}, \ldots, q_{k}\right\}$, define

$$
r(Q)=\operatorname{lcm}\left(q_{j}-q_{i}\right)_{1 \leq i<j \leq k} .
$$

Let $k$ be an integer greater than 1 . Let $R(k)$ be the smallest integer $\rho$ for which there exists a set of $k$ primes for which $r(Q)=\rho$, and $R^{*}(k)$ the smallest one for which there exists an infinity of such $Q$ (under the $k$-tuple conjecture). Clearly, $R(k) \leq R^{*}(k)$. We have proved $R(8)=R^{*}(8)=7$ !. We briefly study the cases $k \leq 12$. We put

$$
D(k)=\prod_{\substack{\varphi\left(p^{\alpha}\right)<k-1 \leq \varphi\left(p^{\alpha+1}\right) \\ p \text { prime }}} p^{\alpha} .
$$

It is not hard to see that, for all $k, D(k)$ divides $R(k)$ and $R^{*}(k)$, thus generalizing (2). So our work is to search for the least multiple of $D(k)$, say $M D(k)$, for which we can find one or an infinity of solutions of $r(Q)=M D(k)$. We use the same method as in Section 4 , and we find cliques $\mathscr{A}=\left(0, a_{2}, \ldots, a_{k}\right)$. Let $\mathscr{P}$ be the set of primes less than or equal to $k$. Depending on whether $\mathscr{A}$ is $\mathscr{P}$-admissible or not, we search for a solution $Q=\left(q_{1}, q_{1}+a_{2}, \ldots, q_{1}+a_{k}\right)$ with $q_{1} \leq k$ or $q_{1}$ unbounded.

The results are shown below in Table 4 . For each $k$, we list $D(k)$ and the ratios $R(k) / D(k)$ and $R^{*}(k) / D(k)$. If $R(k)=R^{*}(k)$, we list the smallest (in reverse 
lexicographic order) admissible solution, else we indicate on the first line a solution for $R(k)$ and on the second line one for $R^{*}(k)$. For $10 \leq k \leq 12$, no $q_{1}$ less than $2^{32}$ gives rise to a solution. I stressed that point with a "?". The proof of $R(k)$ being the right one is missing, since we are not sure that the $k$-tuple conjecture is true.

Let us look at the complexity of the first phase of our algorithm. Suppose we are searching for a set $Q$ of $k$ primes such that $r(Q)=\rho, \rho$ any integer. We have to perform at most $\frac{(k-1)(k-2)}{2}$ verifications for each $k-1$ subset of even divisors of $\rho$. The total number of operations is thus less than

$$
N_{k}(\rho)=\left(\begin{array}{c}
d\left(\frac{\rho}{2}\right) \\
k-1
\end{array}\right) \frac{(k-1)(k-2)}{2} .
$$

Since $k \ll d\left(\frac{\rho}{2}\right)$, we can evaluate

$$
N_{k}(\rho) \sim \frac{d\left(\frac{\rho}{2}\right)^{k-1}}{2(k-3) !}
$$

This estimate looks bad, and fortunately my program achieved a better running time. The sets in Tables 1, 2, 3 were found on a GOUPIL-G4 (IBM-PC compatible), using a few minutes CPU time, and those of Tables 4 on a VAX 11/785, at INRIA**. It took 20 minutes CPU time to find the cliques for $k=12$. The rather exhausting search for the $q_{1}$ 's in Table 3 was done on the VAX, using roughly three hours and a half CPU time to find the largest one (looking through one residue class modulo 210).

TABLE 3. Admissible $S$-sets

\begin{tabular}{|rrrrrrrr|}
\hline$q_{1}$ & $a_{2}$ & $a_{3}$ & $a_{4}$ & $a_{5}$ & $a_{6}$ & $a_{7}$ & $a_{8}$ \\
\hline 11 & 6 & 8 & 12 & 18 & 20 & 36 & 48 \\
23 & 6 & 8 & 18 & 20 & 24 & 36 & 48 \\
11 & 6 & 12 & 18 & 20 & 30 & 36 & 48 \\
11 & 6 & 12 & 18 & 30 & 36 & 42 & 48 \\
23 & 6 & 18 & 20 & 24 & 30 & 36 & 48 \\
20625569 & 12 & 18 & 20 & 24 & 30 & 48 & 60 \\
11 & 12 & 18 & 20 & 30 & 36 & 48 & 60 \\
19459369 & 12 & 18 & 24 & 28 & 30 & 42 & 48 \\
26669 & 12 & 18 & 24 & 30 & 42 & 48 & 60 \\
8573401 & 12 & 18 & 28 & 30 & 36 & 42 & 48 \\
11 & 12 & 18 & 30 & 36 & 42 & 48 & 60 \\
19389079 & 12 & 24 & 28 & 30 & 40 & 42 & 48 \\
2647 & 12 & 24 & 30 & 36 & 40 & 42 & 60 \\
2647 & 12 & 24 & 30 & 36 & 42 & 60 & 72 \\
174729589 & 12 & 24 & 30 & 40 & 42 & 48 & 60 \\
419 & 12 & 24 & 30 & 42 & 48 & 60 & 72 \\
2647 & 12 & 24 & 36 & 42 & 60 & 72 & 84 \\
419 & 12 & 24 & 42 & 48 & 60 & 72 & 84 \\
31 & 12 & 28 & 30 & 36 & 40 & 42 & 48 \\
28082191 & 12 & 30 & 36 & 40 & 42 & 48 & 60 \\
11 & 12 & 30 & 36 & 42 & 48 & 60 & 72 \\
23 & 18 & 20 & 24 & 30 & 36 & 48 & 60 \\
\hline & & & & & & &
\end{tabular}

**Institut National de Recherche en Informatique et en Automatique, Domaine de Voluceau, Rocquencourt, B. P. 105, F-78153 Le Chesnay CEDEX, France. 
TABLE 4. Summary of results

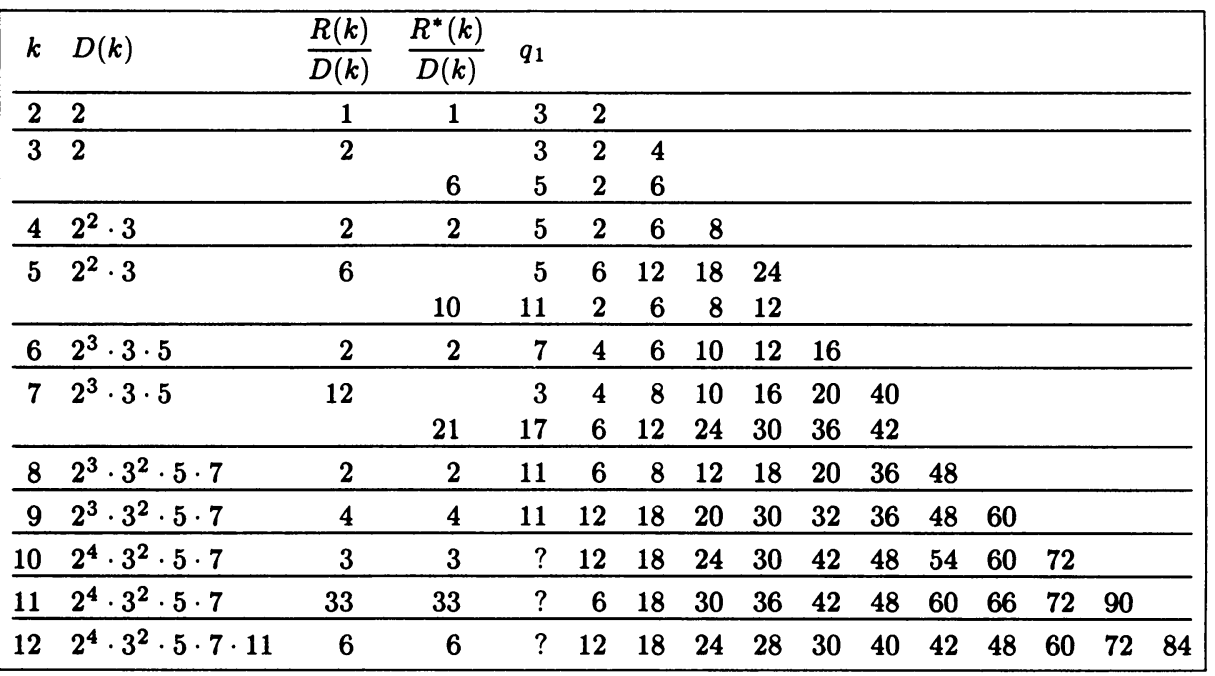

Acknowledgment. I want to thank J. P. Massias, who first told me about using graphs to solve some number theory problems, and Professor J. L. Nicolas for his help while writing this article.

Département de Mathématiques

Université de Limoges

123 Avenue Albert Thomas

F-87060 Limoges Cedex, France

1. E. BALAS \& C. S. YU, "Finding a maximum clique in an arbitrary graph," SIAM J. Comput., v. 15, 1986, pp. 1054-1068.

2. G. H. HARDY \& J. E. LitTlewood, "Some problems of 'Partitio Numerorum'; III: On the expression of a number as a sum of primes," Acta Math., v. 44, 1923, pp. 1-70.

3. D. R. HEATH-BROWN, "The divisor function at consecutive integers," Mathematika, v. 31, 1984, pp. 141-149.

4. D. E. KNUTH, "Sorting and Searching", in The Art of Computer Programming, vol. III, Addison-Wesley, Reading, Mass., 1973.

5. I. RICHARDS, "On the incompatibility of two conjectures concerning primes; A discussion of the use of computers in attacking a theoretical problem," Bull. Amer. Math. Soc., v. 80, 1974, pp. 419-438.

6. C. A. SPIRO, The Frequency with Which an Integral-Valued, Prime-Independent, Multiplicative or Additive Function of $n$ Divides a Polynomial Function of $n, \mathrm{Ph}$. D. Thesis, University of Illinois, Urbana-Champaign, 1981. 